\title{
PATIENT SAFETY ATTITUDE AMONG HEALTH CARE PROVIDERS IN FAMILY HEALTH CARE CENTERS IN CAIRO GOVERNORATE
} By

\author{
MOHSEN ABDALHAMED GADALLAH ${ }^{1}$, HASNAA ABDALAAL ABOUSEIF ${ }^{1}$, DINA \\ NABIH KAMEL BOULOS ${ }^{1}$ AND HANAA HASSAN ABDALLAH ELHARONI ${ }^{2}$ \\ Department of Community, Environmental and Occupational Medicine, Faculty of \\ Medicine, Ain Shams University ${ }^{1}$, Cairo 11566 and Specialist in Family Medicine, \\ Ministry of Health and Population ${ }^{2}$, Cairo, Egypt

\section{Abstract}

Two hundred and eighty Health care; physicians, nurses and technicians were included in the study, 133 (47.5) from $6^{\text {th }}$ discrete HCF, $54(19.3 \%)$ from Duwaiqa and $93(33.2 \%)$ from Al Haggana. Ages ranged between 22 and 59 years $(35.14 \pm 10.13)$, years of experience in the study group ranged between 1 and 35 years $(8.72 \pm 8.18)$ and years of work in group ranged between $1 \& 40$ years $(10.43 \pm 8.33)$. Doctors and nurses were males $(72.55 \%) \&$ females $(86 \%)$ respectively while technicians were mostly males $(60 \%)$. The teamwork climate score was $3.98 \pm 0.87$, $64.0 \%$ answered high or very high, $16.4 \%$ answered inadequate and $18 \%$ had answered few or very few. Safety climate score was $3.61 \pm 0.63,49.28 \%$ had high or very high score, $17.14 \%$ answered inadequate and $32.15 \%$ had answered few or very few. Job satisfaction score was $3.91 \pm 0.80,32.15 \%$ had answered few or very few, $17.14 \%$ answered inadequate and $49.28 \%$ answered high or very high. Stress recognition score was $3.61 \pm 0.79,25 \%$ had answered few or very few, $28.6 \%$ answered inadequate and $45.7 \%$ answered high or very high. Perception of management score was $3.48 \pm 0.80,23.2 \%$ had answered few or very few, $17.8 \%$ answered inadequate and $57.6 \%$ answered high or very high. Working condition score was $3.51 \pm 0.84,46.8 \%$ had answered few or very few, $17.1 \%$ answered inadequate and $35.7 \%$ answered high or very high. A significant difference regarding team work score, safety climate score, perception of management score, working condition score with highest value in doctors and lowest in technicians. On the other hand no significant difference was detected regarding job satisfaction score and stress recognition score.

A significant difference regarding team work score, safety climate score and perception of management score with high values among older groups. No significant difference was detected regarding job satisfaction score, stress recognition score and working condition score. Also, a significant difference regarding team work score and safety climate score with higher value in males. Significant difference regarding job satisfaction score and working condition score was detected with higher value in females. No significant difference was detected regarding stress recognition score and perception of management scores.

Key words: Egypt, Family Health Care Centers. Patient Safety, Health Care Providers,

\section{Introduction}

Patient safety is defined as avoidance and prevention of patient injuries or adverse events resulting from the processes of health care delivery. Various definitions of patient safety have been published, and probably the shortest description is 'to do no harm to patients' (Gaal et al, 2011). The safety culture of an organization acts as a guide as to how employees will behave in the workplace. Of course their behavior influenced or determined by what behaviors were rewarded and acceptable within work-place (Glendon et al, 2014).

Safety culture is defined as the collective product of individual and group values, attitudes and patterns of behaviors in safety performance. Rall et al. (2001) stated that "Patient safety" and "errors in medicine" are issues gaining more and more prominence in the eyes of the public. According to newer studies, errors in medicine are among the ten major causes of death in association with the whole area of health care. They added that the overall though, the most important strategy towards a long- 
term improvement in patient safety will be a change of "culture" throughout the entire health care system. The "culture of blame" focused on individuals should be replaced by a "safety culture", that sees errors and critical incidents as a problem of the whole organization. The acceptance of human fallability and an open-minded nonpunitive analysis of errors in the sense of a "preventive and proactive safety culture" should lead to solutions at the systemic level. This change in culture can only be achieved with a strong commitment from the highest levels of an organization. Patient safety must have the highest priority in the goals of the institution: "Primum nihil nocere"--"First, do not harm". The characteristics of a strong and proactive safety culture include the commitment of the leadership to discuss and learn from errors, to document and improve patient safety, to encourage and practice teamwork, to spot potential hazards, to use systems for reporting and analyzing adverse events and to celebrate workers as heroes improving safety rather than as villains committing errors (Wideman et al, 2005).

Errors and adverse events are common in the outpatient setting because most health care is delivered in the ambulatory setting, providers and researchers are interested in improving patient safety for outpatients. Research in health care and other safety-critical environments, such as the aviation industry, indicates that understanding the systems involved in the delivery of services is important for developing interventions to prevent errors. Attitudes of individuals within organizations may provide insight into how systems function and how they may be improved (Hammons et $a l$, 2003). Health care errors and consequent adverse events are a leading cause of death and injury in the United States, even though methods to prevent many of these errors exist (Gandhi et al, 2003).

A cross sectional study done in 35 primary health care centers in Egyptian
Governorates to measure attitudes of health personnel towards patient safety, and to determine how the concept of patient safety varies between maternal health centers and types of healthcare personnel. Study participants comprised all managers, physicians, nurses, pharmacists, and technicians. The overall mean for all questions and respondents were $3.89 \pm 0.59$. The safety climate mean was $3.64 \pm 0.67$. The percentage of respondents viewing the safety climate as positive was $36 \%$. Only $7 \%$ of respondents had received feed-back after referral of a case of severe pre-eclampsia. The concept of patient safety in the centers studied is not as strong as desirable for the provision of reliable health care. The culture is one of a penalizing nature with suppressed error reporting, lack of proper communication, and feedback failure (Nabhana et al, 2007).

The Aim of the work was to measure attitude of health care providers towards patient safety culture in primary health care centers in Nasr City in Cairo, and To determine different factors affecting patient safety culture.

\section{Subjects, Materials and Methods}

This descriptive cross-sectional study was conducted in three randomly chosen primary governmental health care units in Cairo in 2013. The study population included all available Health care providers (Physicians, Nurses and Technicians) working for more than one year in these three centers. The inclusion criteria: 1males and females, 2- full and part time staff, and 3- working for at least one year prior to the study.

Sampling and study population: For determining the sample size, STATA program version 10 was used, assuming power $80 \%$, CI $95 \%$ the worst positive safety score was $25 \%$ among physicians after Burford et al. (2013) and the worst acceptable frequency was $18 \%$. The least calculated sample size was 280 . Although 324 subjects were asked to participate in the 
study, only 287 accepted to participate. Response rate $=287 / 324 \times 100=88.6 \%$.

Safety attitudes questionnaire ambulatory: A self-administered questionnaire was used for physicians and an interviewing questionnaire to nurses and technicians. The questionnaire is adopted from "The Safety Attitudes Questionnaire Ambulatory Version by the University of Texas at Austin 2003". This questionnaire is designed to assess healthcare provider attitudes about issues relevant to patient safety (often called safety Climate or safety culture). It measures areas of patient safety culture including knowledge and attitude. It was in English and was translated into Arabic with back translation to assure the appropriate meaning.

The SAQ elicits caregiver attitudes through the six factors analytically derived the client scales: teamwork climate; safety climate; job satisfaction; perceptions of management; working conditions; and stress recognition (Relihan et al, 2009)

The SAQ is a questionnaire with 30 items and demographics information (the age, sex, experience and nationality). The questionnaire took approximately 10 to 15 minutes to complete. Each of the 30 items is answered using a five-point Likert scale (Disagree Strongly, Disagree Slightly, Neutral, Agree Slightly, Agree Strongly). Some items are negatively worded. Each version of the SAQ in the current study includes a "Collaboration and Communication" section, where respondents are asked to indicate the quality of collaboration and communication they have experienced with each of the types of providers in their clinical area using a five-point Likert scale; Very Low, Low, Adequate, High, Very High (Zaghloul, 2001).

Pilot study: Twenty questionnaires were distributed to health care workers in Mahkama Primary Health Care Unit before conducting the study.

Ethical consideration: 1. The informed oral consent was obtained from all partici- pants and confidentiality of the data was assured. 2. To ensure confidentiality of data questionnaire was anonymous. 3. Approval from Ain Shams University Ethical Review Committee and from the Public Health Directorate was obtained before the starting this study.

Data management and processing: Data was collected, revised, coded and PC statistical analysis using SPSS version 12.

The Likert scale ( 1 = Disagree Strongly, 2 = Disagree Slightly, 3= Neutral, $4=$ Agree Slightly, $5=$ Agree Strongly) was used to score SAQ items. Negatively worded items were reversely scored so that their valence matched the positively worded items.

Total score of the six factors climate scales: teamwork climate; safety climate; job satisfaction; perceptions of management; working conditions; and stress recognition were calculated by summating the response of their related questions. Each scale includes number of questions: Team work climate scale ( $\mathrm{n}=6$ questions), Safety climate scale $(n=7)$, Job satisfaction scale $(n=5)$, Stress recognition scale $(n=4)$, Perception of management scale $(n=4)$, Working condition scale $(n=4)$.

Total score for each safety attitude scales was calculated by averaging the scores of its subscales. The scores ranged from 1 to 5 , and then these score were classified into 4 categories as follow; $\square 4$ most favorable attitudes, $\leq 4$ somewhat favorable to slightly favorable, $\leq 3$ somewhat unfavorable attitudes 2 and 1 reveals very unfavorable attitudes.

Descriptive statistics and graphical displays were conducted to describe sample population. Frequency tables were utilized to describe nominal and ordinal variables; continuous variables were described using means, standard deviations and range. Chisquare test compared the nominal and the ordinal variables. The student's t-test and ANOVA test were used to compare means of continuous variables for groups. 


\section{Results}

All available health care providers [Physicians (215), Nurses (50) and Technicians (15)] working for more than one year in three randomly chosen primary health care centers were included in the study. Details were given in tables (1 to 24).

Table 1: Age of participants in study group, years of experience and years of work

\begin{tabular}{|c|c|c|c|c|}
\hline Variant & Min & Max & Mean & SD \\
\hline Age (years) & 22.00 & 59.00 & 35.14 & 10.13 \\
\hline Years of experience & 1.00 & 35.00 & 8.72 & 8.18 \\
\hline Years of work & 1.00 & 40.00 & 10.43 & 8.33 \\
\hline
\end{tabular}

Table 2: Age of health care staff groups

\begin{tabular}{|c|c|c|c|c|}
\hline Variant & Physicians $(\mathrm{n}=215)$ & Nurses $(\mathrm{n}=50)$ & Technicians $(\mathrm{n}=15)$ & $\begin{array}{c}\text { Total } \\
(\mathrm{n}=280)\end{array}$ \\
\cline { 2 - 4 } & $\mathrm{N}(\%)$ & $\mathrm{N}(\%)$ & $\mathrm{N}(\%)$ & $1(6.6 \%)$ \\
\hline$<30$ & $52(24.76 \%)$ & $26(52 \%)$ & $8(53.3 \%)$ & $105(37.5 \%)$ \\
\hline $30-44$ & $86(40 \%)$ & $11(22 \%)$ & $5(33.3 \%)$ & $83(29.6 \%)$ \\
\hline $45-54$ & $73(33.9 \%)$ & $5(10 \%)$ & $1(6.6 \%)$ & $13(4.6 \%)$ \\
\hline$\geq 55$ years & $4(1.8 \%)$ & $8(16 \%)$ & & \\
\hline
\end{tabular}

Table 3: Sex \& Marital status of group $(n=280)$

\begin{tabular}{|l|c|c|}
\hline Sex: & No. & $\%$ \\
\hline Male & 75 & $26.8 \%$ \\
\cline { 2 - 3 } Female & 205 & $73.2 \%$ \\
\hline Marital status & 103 & $36.8 \%$ \\
\hline Single & 151 & $53.9 \%$ \\
\hline Married & 24 & $9.6 \%$ \\
\hline Divorced or Widowed
\end{tabular}

Table 4: Sex of health care staff groups

\begin{tabular}{|c|c|c|c|c|}
\hline Sex & Physicians $(\mathrm{n}=215)$ & Nurses $(\mathrm{n}=50)$ & Technicians $(\mathrm{n}=15)$ & Total \\
\hline Male & $59(27.44 \%)$ & $7(14 \%)$ & $9(60 \%) ;$ & $75(26.78 \%)$ \\
\hline Female & $156(72.55 \%)$ & $43(86 \%)$ & $6(40 \%)$ & $205(73.21 \%)$ \\
\hline
\end{tabular}

Table 5: Specialties of medical staff

\begin{tabular}{|l|c|c|}
\hline Specialties & $\mathrm{N}=280$ & $\%$ \\
\hline Family physicians & 78 & 27.9 \\
\hline Dentists & 50 & 17.9 \\
\hline Nursing staff & 46 & 16.4 \\
\hline Pediatrics & 36 & 12.8 \\
\hline Pharmacists & 28 & 10.0 \\
\hline Technicians & 16 & 5.7 \\
\hline Obstetrics \& Gynecologists & 13 & 4.7 \\
\hline Physiotherapists & 13 & 4.6 \\
\hline
\end{tabular}

Table 6: Distribution among health care facilities

\begin{tabular}{|c|c|c|}
\hline Variant & $\mathrm{N}=280$ & $\%$ \\
\hline Sixth District & 133 & 47.5 \\
\hline Duwaiqa & 54 & 19.3 \\
\hline Haggana & 93 & 33.2 \\
\hline
\end{tabular}

Table 7: Employment status of group

\begin{tabular}{|c|c|c|}
\hline Variant & $\mathrm{N}=280$ & $\%$ \\
\hline Full Time & 235 & 84.2 \\
\hline Part Time & 45 & 15.8 \\
\hline
\end{tabular}


Table 8: How group feel about different personals in health facilities

\begin{tabular}{|l|c|c|}
\hline Workers & Mean & SD \\
\hline Physicians & 3.83 & 0.11 \\
\hline Nursing staff & 4.26 & 0.99 \\
\hline Nursing management & 4.74 & 1.28 \\
\hline Resident doctors & 4.17 & 0.82 \\
\hline Director of center & 4.75 & 0.99 \\
\hline Receptionists & 3.13 & 1.99 \\
\hline Nutritionists & 2.81 & 1.33 \\
\hline
\end{tabular}

Table 9: Teamwork Climate scores

\begin{tabular}{|l|c|c|}
\hline \multicolumn{1}{|c|}{ Variant } & Mean & SD \\
\hline I have the support I need from other personnel to care for patients/clients & 4.81 & 1.15 \\
\hline The staff in my area work as a well-coordinated team & 3.14 & 0.59 \\
\hline Total score & 3.98 & 0.87 \\
\hline
\end{tabular}

Table 10: Answer frequencies in team work climate questions

\begin{tabular}{|c|c|c|}
\hline Score & Frequency $\mathrm{n}=276$ & $\%$ \\
\hline High \& v. high & 179 & 64.9 \\
\hline Inadequate & 46 & 16.7 \\
\hline Very few \& few & 51 & 18.4 \\
\hline
\end{tabular}

Table 11: Safety climate scores

\begin{tabular}{|l|c|c|}
\hline \multicolumn{1}{|c|}{ Variant } & Mean & SD \\
\hline I would feel safe being treated here as a patient/client & 3.14 & 0.94 \\
\hline I am encouraged by colleagues to report any patient/client safety concerns I may & 4.08 & 0.32 \\
\hline Total score & 3.61 & 0.63 \\
\hline
\end{tabular}

Table 12: Answer frequencies in safety climate questions

\begin{tabular}{|l|c|c|}
\hline Score & Frequency $\mathrm{n}=276$ & $\%$ \\
\hline High \& v. high & 138 & 50 \\
\hline Inadequate & 48 & 17.39 \\
\hline Very few \& few & 90 & 32.61 \\
\hline
\end{tabular}

Table 13: Job satisfaction scores

\begin{tabular}{|c|c|c|}
\hline Variant & Mean & SD \\
\hline Mv health service is a good place to work & 3.96 & 0.79 \\
\hline I am proud to work here & 3.86 & 0.82 \\
\hline Total score & 3.91 & 0.80 \\
\hline
\end{tabular}

Table 14: Answer frequencies in job satisfaction questions

\begin{tabular}{|l|c|c|}
\hline Score & Frequency $\mathrm{n}=276$ & $\%$ \\
\hline High \& $\mathrm{v}$. high & 138 & 50 \\
\hline Inadequate & 48 & 17.39 \\
\hline Very few \& few & 90 & 32.61 \\
\hline
\end{tabular}

Table 15: Stress recognition scores

\begin{tabular}{|l|c|c|}
\hline \multicolumn{1}{|c|}{ Variant } & Mean & SD \\
\hline Fatigue impairs my performance during emergency situa- & 3.71 & 0.8 \\
\hline I am more likely to make errors in tense or hostile situa- & 3.52 & 0.79 \\
\hline Total score & 3.61 & 0.79 \\
\hline
\end{tabular}

Table 16: Answer frequencies in Stress recognition questions

\begin{tabular}{|l|c|c|}
\hline Score & Frequency $\mathrm{n}=278$ & $\%$ \\
\hline High \& v. high & 128 & 46.04 \\
\hline Inadequate & 80 & 28.78 \\
\hline Very few \& few & 70 & 25.18 \\
\hline
\end{tabular}


Table 17: Perception of management scores

\begin{tabular}{|l|c|c|}
\hline Variant & Mean & SD \\
\hline My administration supports my daily efforts & 3.44 & 0.84 \\
\hline Levels of staffing in my area sufficient to handle number of patients/clients & 3.52 & 0.76 \\
\hline Total score & 3.48 & 0.8 \\
\hline
\end{tabular}

Table 18: Answer frequencies in Perception of management questions

\begin{tabular}{|l|c|c|}
\hline Score & Frequency $\mathrm{n}=276$ & $\%$ \\
\hline High \& v. high & 161 & 58.31 \\
\hline Inadequate & 50 & 18.14 \\
\hline Very few \& few & 65 & 23.55 \\
\hline
\end{tabular}

Table 19: Working condition scores

\begin{tabular}{|c|c|c|}
\hline \multicolumn{1}{|c|}{ Variant } & Mean & SD \\
\hline This health service does a good job training new personnel & 3.57 & 0.87 \\
\hline All necessary data for diagnostic \&therapeutic decisions routinely available to clinical staff & 3.43 & 0.82 \\
\hline Total score & 3.51 & 0.84 \\
\hline
\end{tabular}

Table 20: Answer frequencies in working condition questions

\begin{tabular}{|l|c|c|}
\hline Score & Frequency $\mathrm{n}=279$ & $\%$ \\
\hline High \& v. high & 100 & 35.85 \\
\hline Inadequate & 48 & 17.20 \\
\hline Very few \& few & 131 & 46.95 \\
\hline
\end{tabular}

Table 21: Comparison of SAQ areas total scores regarding different jobs

\begin{tabular}{|l|c|c|c|c|}
\hline Variant & Physicians & Nurses & Technicians & $\mathrm{P}$ \\
\hline Team work scores & $3.96 \pm 0.64$ & $3.87 \pm 0.74$ & $3.68 \pm 0.96$ & $0.02^{*}$ \\
\hline Safety climate score & $4.18 \pm 0.42$ & $3.85 \pm 0.89$ & $3.44 \pm 0.56$ & $0.001^{* *}$ \\
\hline Job satisfaction scores & $4.03 \pm 0.56$ & $3.93 \pm 0.76$ & $3.89 \pm 0.59$ & 0.09 \\
\hline Stress recognition scores & $3.89 \pm 0.93$ & $3.75 \pm 0.45$ & $3.69 \pm 0.68$ & 0.07 \\
\hline Perception of management & $3.64 \pm 0.78$ & $3.43 \pm 0.54$ & $3.46 \pm 0.78$ & $0.03^{*}$ \\
\hline Working condition scores & $3.76 \pm 0.59$ & $3.55 \pm 0.88$ & $3.38 \pm 0.63$ & $0.01^{*}$ \\
\hline
\end{tabular}

Table 22: Comparison of SAQ areas total scores regarding different age groups

\begin{tabular}{|l|c|c|c|c|c|}
\hline Scores & Less than & $30-44$ & $45-54$ & 55 or & $\mathrm{P}$ \\
\hline Team work & $2.92 \pm 1.02$ & $3.87 \pm 0.74$ & $3.96 \pm 0.64$ & $3.68 \pm 0.9$ & $0.03^{*}$ \\
\hline Safety climate & $3.14 \pm 0.56$ & $3.85 \pm 0.89$ & $3.97 \pm 0.72$ & $4.18 \pm 0.4$ & $0.002^{* *}$ \\
\hline Job satisfaction & $2.03 \pm 1.26$ & $3.93 \pm 0.76$ & $3.89 \pm 0.59$ & $4.23 \pm 0.5$ & 0.09 \\
\hline Stress recognition & $3.53 \pm 0.77$ & $3.75 \pm 0.45$ & $3.69 \pm 0.68$ & $3.89 \pm 0.9$ & 0.07 \\
\hline Perception of management & $3.64 \pm 0.78$ & $3.43 \pm 0.54$ & $3.46 \pm 0.78$ & $4.32 \pm 0.9$ & $0.001^{*}$ \\
\hline Working condition & $3.43 \pm 0.32$ & $3.55 \pm 0.88$ & $3.48 \pm 0.63$ & $3.66 \pm 0.5$ & 0.11 \\
\hline
\end{tabular}

Table 23: Comparison of SAQ areas total scores regarding sexes

\begin{tabular}{|l|c|c|c|}
\hline Scores & Males & Females & $\mathrm{P}$ \\
\hline Team work & $3.97 \pm 0.96$ & $3.89 \pm 0.44$ & $0.05^{*}$ \\
\hline Safety climate & $3.88 \pm 0.42$ & $3.85 \pm 0.93$ & $0.05^{*}$ \\
\hline Job satisfaction & $3.23 \pm 0.86$ & $4.43 \pm 0.66$ & $0.005^{* *}$ \\
\hline Stress recognition & $3.87 \pm 0.84$ & $3.55 \pm 0.85$ & 0.09 \\
\hline Perception of management & $3.54 \pm 0.75$ & $3.53 \pm 0.68$ & 0.351 \\
\hline Working condition & $3.36 \pm 0.78$ & $4.25 \pm 0.96$ & $0.001^{* *}$ \\
\hline
\end{tabular}


Table 24: Comparison between study groups regarding team work score.

\begin{tabular}{|l|c|c|c|c|}
\hline \multicolumn{1}{|c|}{ team work } & Doctors & Nurses & Technicians & Chi square test \\
\hline A few & $20(9.3 \%)$ & $2(4 \%)$ & $1(6.7 \%)$ & 23.139 \\
\hline Inadequate & $20(9.3 \%)$ & $2(4 \%)$ & $5(33.3 \%)$ & \\
\hline High \& v. high & $175(81.3 \%)$ & $46(92 \%)$ & $9(60 \%)$ & $0.003 * *$ \\
\hline
\end{tabular}

Table 25: Comparison between groups regarding job satisfaction score

\begin{tabular}{|l|c|c|c|c|}
\hline \multicolumn{1}{|c|}{ job satisfaction } & Doctors & Nurses & Technician & Chi square test \\
\hline A few & $16(7.5 \%)$ & $9(18 \%)$ & $1(6.7 \%)$ & 11.288 \\
\hline Inadequate & $17(7.9 \%)$ & $4(8 \%)$ & 0 & \\
\hline High \& v. high & $179(83.2 \%)$ & $37(74 \%)$ & $14(93.3 \%)$ & 0.322 \\
\hline
\end{tabular}

\section{Discussion}

Quality and safety issues in healthcare continue to receive a growing amount of attention, as role of patient safety culture is regarded as important for patient safety. A positive patient safety attitude is the guide many behaviors of healthcare professionals towards viewing safety as one of the highest priorities (Fujita et al, 2013).

In the present study, two hundred and eighty health care providers (Physicians, Nurses and Technicians) working for more than one year in three randomly chosen primary health care centers were included in the study. $6^{\text {th }}$ discrete facility included 133 (47.5\%) medical staff personal, Duwaiqa included $54(19.3 \%)$ medical staff personnel and Al Haggana included 93 (33.2\%) medical staff personnel. In the present study, 280 questionnaires gave response rate of $88.6 \%$. Ages ranged between 22 and 59 years $(35.14 \pm 10.13)$. Years of experience ranged between one and $35(8.72 \pm 8.18)$ and years of work ranged between $1 \& 40$ years $(10.43 \pm 8.33)$.

Thomas et al. (2003) reported a response rate of $58 \%$ from 320 participants (90 physicians and 230 nurses). The physicians mean age was 47 years $(\mathrm{SD} \pm 10)$, of whom 16 with mean $(\mathrm{SD} \pm 10)$ years of working experience. The nurses' mean age was 39 years $(\mathrm{SD} \pm 9)$ years, of whom 9 with mean $(\mathrm{SD} \pm 7)$ of critical care experience. The physicians were predominantly males $(86 \%)$, and the nurses were predominantly female (92\%).
Ages of participants ranged between 3044 years $(37.5 \%), 45-54(29.6 \%)$ and, less than 30 years $(28.2 \%)$. Only $4.6 \%$ of the sample were 50 years old or more. The majority of medical staff included in the study were females $(73.2 \%)$. One hundred and three subjects (36.7\%) were single, 151 $(53.5 \%)$ were married, and $24(9.6 \%)$ were divorced or widowed.

In the present study, health care providers in the 30 to 44 year old age group were more likely than were younger to participate contrasts with previous findings of higher response rates for younger health care workers. Cull et al. (2005) in USA tracked response rates across time for surveys of pediatricians, to explore whether response bias was present for these surveys, and to examine whether response bias increases with lower response rates. The concluded that while response biases favoring women, young physicians, and nonspecialty-fellow members were found across the $52-81 \%$ response rates examined in this study, the amount of bias was minimal for these factors that could be tested. At least for surveys of pediatricians, more attention should be devoted by investigators to assessments of response bias rather than relying on response rates as a proxy of response bias. Armstrong et al. (2009) in USA mentioned that nursing programs respond to vital initiatives such as Quality and Safety Education for Nurses (QSEN), nursing faculty will discover important shared values exist between competency- 
based curricular models and the latest call for stronger foci on safety and quality. They concluded that nursing faculty whose teaching is focused in a competency-based curriculum was well positioned to respond to the call to integrate QSEN competencies into their curricula. These differences might be due to different cultures as well as the speculation as Cull et al. (2005) dealt with pediatricians and Armstrong et al. (2009) dealt with nurses in contrast to the current study dealt with all professional medical groups.

In the present study, two hundred and thirty five $(84.2 \%)$ subjects were working full time and $45(15.85 \%)$ were working part time. De Smedt et al. (2006) in Belgian describes primary care professionals' self-reported attitudes towards evidencebased practice (EBP), attention to information sources, perceptions of the barriers to EBP and strategies to improve insight in EBP and patient care They concluded that the most appropriate method for actual implementation of evidence-based practice at all levels of health care is to provide summaries of evidence, easily understandable protocols and web-based databases accessible from the working environment. Students should not only learn the skills related to EBP, but should be able to integrate knowledge effectively in the clinical setting and routine care. Above all, their supervisors themselves need to evolve from 'experience-based' to evidence-based practice'. Braithwaite et al. (2011) found that among all groups of health care staff the most common age group was 45-59 years, followed by $30-44$ years. They added that $54 \%$ had a full time work which was lower than the present results. They concluded that differences between safety specialists' and workforce groups' beliefs about how to improve patient safety may impede the successful implementation of the patientsafety programs.

In the present study, the teamwork climate score was $3.98 \pm 0.87$, safety climate score was $3.61 \pm 0.63$, job satisfaction score was $3.91 \pm 0.80$, stress recognition score was $3.61 \pm 0.79$, perception of management score was $3.48 \pm 0.80$, and working condition score was $3.51 \pm 0.84$. Callen et al. (2009) in Australia found that the scale achieving the highest mean score was Job Satisfaction (3.90). This was followed by the centrally important scale in the questionnaire Safety Climate $($ mean $=3.88)$ and Teamwork climate $($ mean=3.82). All these scores were in the upper section of 34 score band indicating that on average respondents were somewhat in agreement that their workplaces possessed such characteristics. Stress recognition scores were also within this upper band (mean $=3.67$ ). Working Conditions were rated at midway in the 3-4 band (mean=3.49) and Perception of Management received a slightly lower rating (mean=3.44). Similar to the present results, none of the mean scores on the six SAQ scales reached the 4-5 band, which indicates stronger agreement, none were below 3 which would reveal unfavorable or negative attitudes. Lehnbom et al. (2014) in Australia examined the evidence regarding the effectiveness of medication reconciliation and review and to improve clinical outcomes in hospitals, the community, and aged care facilities, They concluded that the evidence demonstrated that medication reconciliation has the potential to identify many medication discrepancies and reduced potential harm, but the impact on clinical outcomes is less clear.

Similarly, medication review could detect medication-related problems in many patients, but evidence of clinical impact is scant. Overall, there was limited evidence that medication reconciliation and medication review processes, as currently performed, significantly improve clinical outcomes, such as reductions in hospital readmissions.

In the current study, comparing SAQ sub-scores in different study groups, a sig- 
nificant difference regarding team work score $(p=0.02)$ with highest value in doctors $(3.96 \pm 0.64)$ and lowest in technicians (3.68 \pm 0.96$)$ was detected. Thomas et al. (2003) reported that teamwork survey results with nurses and physicians working together who viewed the success of their teamwork with each other differently. Physicians reported more favorable perceptions of teamwork. They demonstrated differences in teamwork climate scale scores between physicians and nurses $(\mathrm{p}=0.001)$, and nurses' scores $(\mathrm{M}=3.23)$ showed less satisfaction with teamwork climate than physicians' scores $(\mathrm{M}=3.74)$. De Fontes and Surbida (2004) also reported that physicians viewed teamwork with nurses more positively than nurses viewed teamwork with physicians. Mills et al. (2008) found that doctors rate teamwork and communication during their work more favorably relative to nurses.

Matziou et al. (2014) in Greece investigated the physician and nursing perceptions regarding communication and collaboration as well as the factors that may influence these activities. They concluded that in everyday practice, both nurses and physicians should acknowledge the importance of their effective communication and they should develop and implement inter-professional teamwork interventions to improve collaboration. Moreover, nurses have to constantly consolidate their role in the decision process and patients' care, especially in countries with limited interprofessional collaboration culture. In addition, factors that improve physicians' attitudes toward collaboration and effective communication should be further explored.

The present study showed highly significant difference regarding safety climate score $(p=0.001)$ with highest value in doctors $(4.18 \pm 0.42)$ and lowest in technicians (3.44 \pm 0.56$)$.

Singer et al. (2009) in USA administered the Patient Safety Climate in Healthcare Organizations survey in 2004-2005 to per- sonnel in a stratified random sample of 92 US hospitals. They found that Differences among and within hospitals suggest that strategies for improving safety climate and patient safety should be tailored for work areas and disciplines and that nurses and technicians perceived less institutional commitment to safety than did physicians may suggest that they are more likely to observe deficiencies in the organizational infrastructure related to patient safety than are physicians

Steinemann et al. (2011) in USA evaluated the impact of a team training curriculum for residents and multidisciplinary trauma team members on team communication, coordination and clinical efficacy of trauma resuscitation. They found that aq relatively brief (four-hour) a human patient simulator (HPS)-based curriculum can improve the teamwork and clinical performance of multidisciplinary trauma teams that include surgical residents. This improvement was evidenced both in simulated and actual trauma settings, and across teams of varying composition. HPS-based trauma teamwork training appears to be an educational method that can impact patient care..

The current study showed a significant difference regarding perception of management score $(\mathrm{p}=0.03)$ with highest value in doctors (3.64 \pm 0.78$)$ and lowest in nurses (3.44 \pm 0.56$)$. Nurses and technicians often spend more time with patients than do physicians (Corser, 2000).

Finlayson et al. (2007) replicated a crossnational study of nurses' perceptions of staffing, work organization and outcomes conducted in more than 700 hospitals in the United States, Canada, England, Scotland, and Germany. They found that the New Zealand nurses report similar shortcomings in their work environment as do the nurses in countries with distinctly different health care systems. While they report similar high levels of competence and good relations between doctors and nurses as the respondents in the other five countries, 
higher numbers of New Zealand nurses 30 years of age or younger report their intention to leave their current jobs. New Zealand nurses also report the highest levels of job related stress, high levels of job dissatisfaction, and more than half report receiving inadequate organizational support. Miró (2010) stated that Spain has universal public health care coverage. He added that despite the high EM caseload and the potential severity of the conditions, training in EM is still unregulated in Spain. However, in April 2009 the Spanish Minister of Health announced the imminent approval of an EM specialty, allowing the first EM resident to officially start in 2011. Spanish emergency physicians look forward to the final approval, which will complete the modernization of emergency health care provision in Spain.

Rainer (2014) reported that speaking up to protect patients was a key ethical and moral mandate for nurses, silence still prevails in many situations. On the basis of concepts of safety culture, generational theory, personal cultural literature, advocacy theory, oppressed group theory, and moral distress theory, the proposed theory identifies primary factors of speaking up: generational, personal culture, and organizational.

The present study showed a significant difference regarding working condition score $(p=0.01)$ with highest value in doctors $(3.76 \pm 0.59)$ and lowest in technicians (3.38 \pm 0.63$)$ was detected. The relatively negative nurse ratings of the dimensions "working conditions" and "perceptions towards the hospital" are unsurprising given that work dissatisfaction and high turnover are well-documented problems in the nursing profession ( $\mathrm{Lu}$ et al, 2005). Inflexible working schedules, overwhelming workloads and an unsupportive health-care environment have been shown to have led to the burnout among nurses.

Dalmolin et al. (2014) identified the frequency and intensity of moral distress ex- perienced by nurses, technicians and nursing assistants who worked in hospitals in the South of Rio Grande do Sul State. They found that nurses and nursing assistants have higher perception of moral distress when compared to nursing technicians. Organizational questions and ways of communication influence lower perception of moral distress. They concluded that Implementation of actions to favor coping, decision making and autonomy exercise from those workers.

The current study showed no significant difference was detected regarding job satisfaction score and stress recognition score.

The technicians, whose primary tasks are examining and analyzing body fluids and cells and who tend to work independently rather than in cooperation with coworkers (Wolf, 1999) gave more negative ratings than did physicians and nurses for the job satisfaction. Huntington et al. (2009) stated that the internet has only recently been used for health research. However, webbased methodologies are increasingly discussed as significant and inevitable developments in research as Internet access and use rapidly increases worldwide. They added that Internet-based technology can support the maintenance of cohorts across multiple countries and jurisdictions to explore factors influencing workforce participation. However, barriers to widespread adoption of web-based approaches include website development costs, the need for fast broadband connection for large data collection instruments, and varying degrees of internet and computer literacy in the nursing and midwifery workforce. They concluded that the development of on-line methods and tools is a major and exciting development in the world of research. Research via the world-wide web can support international collaborations across borders and cultures.

Tuckett et al. (2014) stated that the shortage of nurses is known and explored nurses' reasons for leaving the profession based 
on responses from an e-newsletter of the Nurses and Midwives e-cohort Study. They reported that by qualitative content analysis of data from email responses $(n=66)$ showed 'Lack of support' as a social work value describing their manager's lack of support, unsupportive relationships within their work group, and a health-care system putting business principles before care resulting in job dissatisfaction and nurse turnover.

\section{Conclusion}

As healthcare becomes more complex, safe patient centered care is directly influenced by the quality of the education that health care professionals receive. Based on a review of the literature, further research to support and guide curricular change and more studies focused on instructional methods are needed.

It is possible to reliably and meaning fully measure caregiver attitudes and perceptions relevant to the safety of healthcare. The use of the SAQ to assess climate in clinical areas will allow valid comparisons between hospitals, patient care areas, and types of caregivers, and tracking of change over time. No doubt, quantitative assessment of organizational safety culture with reliable and valid questionnaire could distinguish features of organizational culture for patient safety across subgroups such as hospitals, professions, management/non-management positions and the units/wards further the understanding of associated characteristics of each subgroup and provide insight into organizational readiness for patient safety improvement. The feedback from clinical incident reviews closes the loop of the review process and creates a platform for necessary changes to be undertaken.

\section{Acknowledgement}

The present authors like to thank the physicians, nurses and technicians who kindly shared in this study. Thanks are also to the Public Health Authorities who kindly allowed and facilitates the work.

\section{References}

Armstrong, GE, Spencerm TS, Lenburg, CB, 2009: Using quality and safety education for nurses to enhance competency outcome performance assessment: a synergistic approach that promotes patient safety and quality outcomes. J. Nurs. Educ. 48, 12:686-93.

Braithwaite, J, Westbrook, MT, Robinson, M, Michael, S, Pirone, C, et al, 2011: Improving patient safety: the comparative views of patient-safety specialists, workforce staff and managers. BMJ Qual. Saf. 20, 5:424-31.

Burford, BJ, Welch, V, Waters, E, Tugwell, P, Moher, D, et al, 2013: Testing the PRISMA-Equity 2012 reporting guideline: the perspectives of systematic review authors. PLoS. One 8, 10:e75122.

Callen, J, Braithwaite, J, Westbrookl, JI, 2009: The importance of medical and nursing sub-cultures in the implementation of clinical information systems. Methods Inf. Med. 48, 2:196-202

Corser, WD, 2000: The contemporary nursephysician relationship: Insights from scholars outside the two professions. Nurs. Outlook 48, 6:263-8.

Dalmolin, GD, Lunardi, VL, Lunardi, GL, Barlem, EL, Silveira, RS, 2014: Nurses, nursing technicians and assistants: Who experiences more moral distress? Rev. Esc. Enferm. USP. 48, 3:521-9

De Fontes, J, Surbida S, 2006: Preoperative safety briefing project. Permanente J. 8:21-7.

De Smedt, A, Buyl, R, Nyssen, M, 2006: Evidence-based practice in primary health care. Stud. Hlth. Technol. Inform. 124:651-6.

Finlayson, M, Aiken, L, Nakarada-Kordic, I, 2007: New Zealand nurses' reports on hospital care: an international comparison. Nurs. Prax. N Z. 23, 1:17-28.

Fujita, S, Seto, K, Ito, S, Wu, Y, Huangm C, 2013: The characteristics of patient safety culture in Japan, Taiwan and the United States. BMC Hlth. Serv. Res. 13:20-9.

Gaal, S, Wolters, R, Verstappen, W, Lankveld, H, van-Weel, C, et al, 2011: Prevalence and consequences of patient safety incidents in general practice in the Netherlands: A retrospective medical record review study. Implement Sci. 6:37-42.

Gandhi, TK, Weingart, SN, Borus, J, et al, 2003: Adverse drug events in ambulatory care. N. Engl. J. Med. 348, 16:1556-64. 
Glendon, AI, McNally, B, Jarvis, A, Chalmers, SL, Salisbury, RL, 2014: Evaluating a novice driver and pre-driver road safety intervention. Accid. Anal. Prev. 64:100-10

Hammons, T, Piland, NF, Small, SD, Hatlie, MJ, Burstin, HR, 2003: Ambulatory patient safety. What we know and need to know. J. Ambul. Care Manage. 26, 1:63-82.

Huntington, A, Gilmour, J, Schluter, P, Tuckett, A, Bogossian, F, et al, 2009: The Internet as a research site: establishment of a web-based longitudinal study of the nursing and midwifery workforce in three countries. J. Adv, Nurs. 65, 6:1309-17.

Lehnbom, EC, Stewart, MJ, Manias, E, Westbrook, JI, 2014: The Impact of Medication Reconciliation and Review on Clinical Outcomes. Ann. Pharmacother. 2014 Jul 21. pii: 1060028014543485 .

Lu, H, While, AE, Barriball, KL, 2005: Job satisfaction among nurses: A literature review. Int. J. Nurs. Stud. 42, 2:211-27.

Matziou, V, Vlahioti, E, Perdikaris, P, Matziou, T, Megapanou, E, et al, 2014: Physician and nursing perceptions concerning interprofessional communication and collaboration. J. Interprof. Care 8:1-8.

Mills, P, Neily, J, Dunn, E, 2008: Teamwork and communication in surgical teams: Implications for patient safety. J. Am. Coll. 206:10712.

Miró, O, 2010: State of emergency medicine in Spain. Int. J. Emerg. Med. 3, 4:219-26.

Rainer, J, 2014: Speaking Up: Factors and Issues in Nurses Advocating for Patients When Patients Are in Jeopardy. J. Nurs. Care Qual. $2014 \mathrm{Jul}$ 31. [Epub ahead of print]

Rall, M, Manser, T, Guggenberger, H, Gaba, DM, Unertl, K, 2001: Patient safety and errors in medicine: development, prevention and analyses of incidents. Anasthesiol.
Intensivmed. Notfallmed. Schmerzther. 36, 6: 321-30.

Relihan, E, Glynn, S, Daly, D, Silke, B, Ryder, S, 2009: Measuring and benchmarking safety culture: application of the safety attitudes questionnaire to an acute medical admissions unit. Ir. J. Med. Sci. 178, 4:433-9.

Singer, SJ, Gaba, DM, Falwell, A, Lin, S, Hayes, J, et al, 2009: Patient safety climate in 92 US hospitals differences by work area and discipline. Med. Care 47, 1:23-31.

Steinemann, S, Berg, B, Skinner, A, DiTulio, A, Anzelon, K, et al, 2011: In situ, multidisciplinary, simulation-based teamwork training improves early trauma care. J. Surg. Educ. 68, 6:472-7

Thomas, EJ, Sexton, JB, Helmreich, RL, 2003: Discrepant attitudes about teamwork among critical care nurses and physicians. Crit. Care Med. 31:956-9.

Tuckett, A, Winters-Chang, P, Bogossian, F, Wood, M, 2014: 'Why nurses are leaving the profession ... lack of support from managers': What nurses from an e-cohort study said. Int. J. Nurs. Pract. Feb 26. doi: 10.1111/ijn.12245.

Wideman, MV, Whittler, ME, Anderson, TM, 2005: Barcode medication administration: Lessons learned from an intensive care unit implementation. In: Advances in Patient Safety: Henriksen, K, Battles, JB, Marks, ES, Lewin, DI, (eds.) Research to Implementation, Vol.3. Rockville (MD): Agency for Healthcare Research and Quality USA.

Wolf, KN, 1999: Allied health professionals and attitudes toward teamwork. J. Allied Hlth. $28,1: 15-20$.

Zaghloul, AA, 2001: Validation of a patient satisfaction scale for primary care settings. J. Egypt. Publ. Hlth. Assoc. 76, 5/6:411-29. 